\title{
JOINTLY OPTIMIZED HIGH-ORDER ERROR FEEDBACK AND REALIZATION FOR ROUNDOFF NOISE MINIMIZAION IN STATE-SPACE DIGITAL FILTERS
}

\author{
Takao Hinamoto, Akimitsu Doi \\ Hiroshima Institute of Technology \\ Hiroshima 731-5193, Japan \\ Emails: hinamoto@ieee.org, doi@cc.it-hiroshima.ac.jp
}

\author{
Wu-Sheng Lu \\ University of Victoria \\ Victoria, BC, Canada V8W 3P6 \\ Email:wslu@ece.uvic.ca
}

\begin{abstract}
The joint optimization problem of high-order error feedback and realization for state-space digital filters to minimize the effects of roundoff noise at the filter output subject to $l_{2}$ scaling constraints is investigated. The problem is converted into an unconstrained optimization problem by using linear-algebraic techniques. The unconstrained optimization problem at hand is then solved iteratively by applying an efficient quasi-Newton algorithm with closed-form formulas for key gradient evaluation. Finally, a numerical example is presented to illustrate the utility of the proposed technique.
\end{abstract}

\section{INTRODUCTION}

The problem of reducing the effects of roundoff noise at the filter output is of critical importance in the implementation of IIR digital filters in fixed-point arithmetic. Error feedback (EF) is found useful for the reduction of finite-word-length (FWL) effects in IIR digital filters, and many EF techniques have been reported in the past [1]-[10]. Alternatively, the roundoff noise can also be reduced by introducing a delta operator to IIR digital filters [11]-[13], or by applying a new structure based on the concept of polynomial operators for digital filter implementation [14]. Another useful approach is to construct the state-space filter structures for the roundoff noise gain to be minimized by applying a linear transformation to statespace coordinates subject to $l_{2}$-scaling constraints [15]-[18]. As a natural extension of the aforementioned methods, efforts have been made to develop new methods that combine EF and state-space realization, for achieving better performance [19][20]. Separately-optimized analytical algorithms have been proposed for state-space digital filters [19]. Jointly-optimized iterative algorithms have also been considered for filters with a general or scalar EF matrix [19]. In [20], a jointly-optimized iterative algorithm has been developed for state-space digital filters with a general, diagonal, or scalar EF matrix using a quasi-Newton method.

This paper investigates the problem of jointly optimizing high-order EF and realization for state-space digital filters to minimize the roundoff noise subject to $l_{2}$-scaling constraints. An iterative technique which relies on an efficient quasi-Newton algorithm [21] is developed. To this end, the constrained optimization problem encountered is converted into an unconstrained optimization problem by using linearalgebraic techniques. The proposed technique is applied to the cases where the high-order EF has diagonal or scalar matrices. A numerical example is presented to illustrate the proposed algorithm and demonstrate its performance.

\section{PROBLEM STATEMENT}

Consider a stable, controllable and observable state-space digital filter $(\boldsymbol{A}, \boldsymbol{b}, \boldsymbol{c}, d)_{n}$ described by

$$
\begin{aligned}
\boldsymbol{x}(k+1) & =\boldsymbol{A} \boldsymbol{x}(k)+\boldsymbol{b} u(k) \\
y(k) & =\boldsymbol{c} \boldsymbol{x}(k)+d u(k)
\end{aligned}
$$

where $\boldsymbol{x}(k)$ is an $n \times 1$ state-variable vector, $u(k)$ is a scalar input, $y(k)$ is a scalar output, and $\boldsymbol{A}, \boldsymbol{b}, \boldsymbol{c}$ and $d$ are real constant matrices of appropriate dimensions.

By taking the quantizations performed before matrix-vector multiplication into account, an finite-word-length implementation of (1) with error feedforward and high-order EF can be obtained as

$$
\begin{aligned}
\tilde{\boldsymbol{x}}(k+1) & =\boldsymbol{A} \boldsymbol{Q}[\tilde{\boldsymbol{x}}(k)]+\boldsymbol{b} u(k)+\sum_{i=1}^{N} \boldsymbol{D}_{i} \boldsymbol{e}(k-i+1) \\
\tilde{y}(k) & =\boldsymbol{c} \boldsymbol{Q}[\tilde{\boldsymbol{x}}(k)]+d u(k)+\boldsymbol{h} \boldsymbol{e}(k)
\end{aligned}
$$

where $\boldsymbol{h}$ and $\boldsymbol{D}_{1}, \boldsymbol{D}_{2}, \cdots, \boldsymbol{D}_{N}$ are referred to as a $1 \times n$ error-feedforward vector and $n \times n$ high-order error-feedback matrices, respectively, and $\boldsymbol{e}(k)=\tilde{\boldsymbol{x}}(k)-\boldsymbol{Q}[\tilde{\boldsymbol{x}}(k)]$.

The coefficient matrices $\boldsymbol{A}, \boldsymbol{b}, \boldsymbol{c}$, and $d$ in (2) are assumed to have exact fractional $B_{c}$-bit representations. The FWL state-variable vector $\tilde{\boldsymbol{x}}(k)$ and the output $\tilde{y}(k)$ all have $B$-bit fractional representations, while the input $u(k)$ is a $\left(B-B_{c}\right)$ bit fraction. The quantizer $\boldsymbol{Q}[\cdot]$ in (2) rounds the $B$-bit fraction $\tilde{\boldsymbol{x}}(k)$ to $\left(B-B_{c}\right)$-bit after the multiplications and additions, where the sign bit is not counted. It is assumed that the roundoff error $\boldsymbol{e}(k)$ can be modeled as a zero-mean noise process with covariance $\sigma^{2} \boldsymbol{I}_{n}$. Subtracting (2) from (1) yields

$$
\begin{aligned}
\Delta \boldsymbol{x}(k+1) & =\boldsymbol{A} \Delta \boldsymbol{x}(k)+\boldsymbol{A} \boldsymbol{e}(k)-\sum_{i=1}^{N} \boldsymbol{D}_{i} \boldsymbol{e}(k-i+1) \\
\Delta y(k) & =\boldsymbol{c} \Delta \boldsymbol{x}(k)+(\boldsymbol{c}-\boldsymbol{h}) \boldsymbol{e}(k)
\end{aligned}
$$

where $\Delta \boldsymbol{x}(k)=\boldsymbol{x}(k)-\tilde{\boldsymbol{x}}(k)$ and $\Delta y(k)=y(k)-\tilde{y}(k)$. By taking the $z$-transform on both sides of (3) and setting $\Delta \boldsymbol{x}(0)=\mathbf{0}$, we have

$$
\begin{aligned}
\Delta Y(z)= & \boldsymbol{H}_{e}(z) \boldsymbol{E}(z) \\
\boldsymbol{H}_{e}(z)= & \boldsymbol{c}\left(z \boldsymbol{I}_{n}-\boldsymbol{A}\right)^{-1}\left(\boldsymbol{A}-\sum_{i=1}^{N} \boldsymbol{D}_{i} z^{-i+1}\right) \\
& +\boldsymbol{c}-\boldsymbol{h}
\end{aligned}
$$


where $\Delta Y(z)$ and $\boldsymbol{E}(z)$ represent the $z$-transforms of $\Delta y(k)$ and $\boldsymbol{e}(k)$, respectively. $\boldsymbol{H}_{e}(z)$ in (4) is written as

$$
\boldsymbol{H}_{e}(z)=\sum_{k=1}^{\infty} \boldsymbol{c}\left(\boldsymbol{A}^{k}-\sum_{i=1}^{N} \boldsymbol{A}^{k-i} \boldsymbol{D}_{i}\right) z^{-k}+\boldsymbol{c}-\boldsymbol{h}
$$

where $\boldsymbol{A}^{i}=\mathbf{0}$ for $i<0$. Next, we define the normalized noise gain $J_{e 1}(\boldsymbol{h}, \boldsymbol{D})=\sigma_{\text {out }}^{2} / \sigma^{2}$ with $\boldsymbol{D}=\left[\boldsymbol{D}_{1}, \boldsymbol{D}_{2}, \cdots, \boldsymbol{D}_{N}\right]$ as

$$
J_{e 1}(\boldsymbol{h}, \boldsymbol{D})=\operatorname{tr}\left[\frac{1}{2 \pi j} \oint_{|z|=1} \boldsymbol{H}_{e}^{*}(z) \boldsymbol{H}_{e}(z) \frac{d z}{z}\right]
$$

Substituting (5) into (6) yields

$$
\begin{aligned}
& J_{e 1}(\boldsymbol{h}, \boldsymbol{D}) \\
&=\operatorname{tr}\left[\boldsymbol{A}^{T} \boldsymbol{W}_{o} \boldsymbol{A}-\sum_{i=1}^{N}\left\{\left(\boldsymbol{A}^{T}\right)^{i} \boldsymbol{W}_{o} \boldsymbol{D}_{i}+\boldsymbol{D}_{i}^{T} \boldsymbol{W}_{o} \boldsymbol{A}^{i}\right\}\right. \\
& \quad+\sum_{i=1}^{N} \sum_{j=1}^{N} \boldsymbol{D}_{i}^{T}\left\{\left(\boldsymbol{A}^{T}\right)^{j-i} \boldsymbol{W}_{o}+\boldsymbol{W}_{o} \boldsymbol{A}^{i-j}\right\} \boldsymbol{D}_{j} \\
&\left.\quad-\sum_{i=1}^{N} \boldsymbol{D}_{i}^{T} \boldsymbol{W}_{o} \boldsymbol{D}_{i}\right]+(\boldsymbol{c}-\boldsymbol{h})(\boldsymbol{c}-\boldsymbol{h})^{T}
\end{aligned}
$$

where $\boldsymbol{W}_{o}$ is the observability Gramian of the filter in (1) that can be obtained by solving the Lyapunov equation

$$
\boldsymbol{W}_{o}=\boldsymbol{A}^{T} \boldsymbol{W}_{o} \boldsymbol{A}+\boldsymbol{c}^{T} \boldsymbol{c} .
$$

Assuming that $\boldsymbol{D}_{1}, D_{2}, \cdots, D_{N}$ are diagonal, (7) can be written as

$$
\begin{aligned}
J_{e 1}(\boldsymbol{h}, \boldsymbol{D})=\operatorname{tr}\left[\boldsymbol{A}^{T} \boldsymbol{W}_{o} \boldsymbol{A}-2 \sum_{i=1}^{N} \boldsymbol{W}_{o} \boldsymbol{A}^{i} \boldsymbol{D}_{i}\right. \\
\left.\quad+\sum_{i=1}^{N} \sum_{j=1}^{N} \boldsymbol{W}_{o} \boldsymbol{A}^{|i-j|} \boldsymbol{D}_{i} \boldsymbol{D}_{j}\right] \\
+(\boldsymbol{c}-\boldsymbol{h})(\boldsymbol{c}-\boldsymbol{h})^{T} .
\end{aligned}
$$

It should be noted that the $l_{2}$-scaling constraints on the statevariable vector $\boldsymbol{x}(k)$ involve the controllability Gramian $\boldsymbol{K}_{c}$ of the filter in (1) which can be computed by solving the Lyapunov equation

$$
\boldsymbol{K}_{c}=\boldsymbol{A} \boldsymbol{K}_{c} \boldsymbol{A}^{T}+\boldsymbol{b} \boldsymbol{b}^{T} .
$$

A different yet equivalent state-space description of (1), $(\overline{\boldsymbol{A}}, \overline{\boldsymbol{b}}, \overline{\boldsymbol{c}}, d)_{n}$, can be obtained via a coordinate transformation $\overline{\boldsymbol{x}}(k)=\boldsymbol{T}^{-1} \boldsymbol{x}(k)$ where

$$
\bar{A}=T^{-1} A T, \quad \bar{b}=T^{-1} b, \quad \bar{c}=c T .
$$

Accordingly, the controllability and observability Gramians for $(\overline{\boldsymbol{A}}, \overline{\boldsymbol{b}}, \overline{\boldsymbol{c}}, d)_{n}$ become

$$
\overline{\boldsymbol{K}}_{c}=\boldsymbol{T}^{-1} \boldsymbol{K}_{c} \boldsymbol{T}^{-T}, \quad \overline{\boldsymbol{W}}_{o}=\boldsymbol{T}^{T} \boldsymbol{W}_{o} \boldsymbol{T}
$$

respectively. The $l_{2}$-scaling constraints are imposed on the state-variable vector $\overline{\boldsymbol{x}}(k)$ so that

$$
\left(\overline{\boldsymbol{K}}_{c}\right)_{i i}=\left(\boldsymbol{T}^{-1} \boldsymbol{K}_{c} \boldsymbol{T}^{-T}\right)_{i i}=1, \quad i=1,2, \cdots, n .
$$

The problem being considered is to design the high-order EF diagonal matrices $\boldsymbol{D}_{1}, \boldsymbol{D}_{2}, \cdots, \boldsymbol{D}_{N}$ as well as the optimal coordinate transformation matrix $\boldsymbol{T}$ that jointly minimize

$$
\begin{array}{r}
J_{e 2}(\boldsymbol{T}, \boldsymbol{D})=\operatorname{tr}\left[\overline{\boldsymbol{A}}^{T} \overline{\boldsymbol{W}}_{o} \overline{\boldsymbol{A}}-2 \sum_{i=1}^{N} \overline{\boldsymbol{W}}_{o} \overline{\boldsymbol{A}}^{i} \boldsymbol{D}_{i}\right. \\
\left.+\sum_{i=1}^{N} \sum_{j=1}^{N} \overline{\boldsymbol{W}}_{o} \overline{\boldsymbol{A}}^{|i-j|} \boldsymbol{D}_{i} \boldsymbol{D}_{j}\right]
\end{array}
$$

subject to $l_{2}$-scaling constraints in (13) where the error feedforward vector $\boldsymbol{h}$ is assumed to be chosen as $\boldsymbol{h}=\overline{\boldsymbol{c}}$.

\section{JOINT OPTIMIZATION OF HIGH-ORDER ERROR FEEDBACK AND REALIZATION}

To deal with (13), we define

$$
\hat{\boldsymbol{T}}=\boldsymbol{T}^{T} \boldsymbol{K}_{c}^{-\frac{1}{2}} .
$$

The $l_{2}$-scaling constraints in (13) can then be written as

$$
\left(\hat{\boldsymbol{T}}^{-T} \hat{\boldsymbol{T}}^{-1}\right)_{i i}=1, \quad i=1,2, \cdots, n .
$$

These constraints are always satisfied if $\hat{\boldsymbol{T}}^{-1}$ assumes the form

$$
\hat{\boldsymbol{T}}^{-1}=\left[\frac{\boldsymbol{t}_{1}}{\left\|\boldsymbol{t}_{1}\right\|}, \frac{\boldsymbol{t}_{2}}{\left\|\boldsymbol{t}_{2}\right\|}, \cdots, \frac{\boldsymbol{t}_{n}}{\left\|\boldsymbol{t}_{n}\right\|}\right] .
$$

Substituting (15) into (14), we obtain

$$
\begin{array}{r}
J(\hat{\boldsymbol{T}}, \boldsymbol{D})=\operatorname{tr}\left[\hat{\boldsymbol{A}}^{T} \hat{\boldsymbol{W}}_{o} \hat{\boldsymbol{A}}-2 \sum_{p=1}^{N} \hat{\boldsymbol{W}}_{o} \hat{\boldsymbol{A}}^{p} \boldsymbol{D}_{p}\right. \\
\left.+\sum_{p=1}^{N} \sum_{q=1}^{N} \hat{\boldsymbol{W}}_{o} \hat{\boldsymbol{A}}^{|p-q|} \boldsymbol{D}_{p} \boldsymbol{D}_{q}\right]
\end{array}
$$

where

$$
\begin{array}{crl}
\hat{\boldsymbol{A}}=\hat{\boldsymbol{T}}^{-T} \boldsymbol{K}_{c}^{-\frac{1}{2}} \boldsymbol{A} \boldsymbol{K}_{c}^{\frac{1}{2}} \hat{\boldsymbol{T}}^{T}, \quad \hat{\boldsymbol{b}}=\hat{\boldsymbol{T}}^{-T} \boldsymbol{K}_{c}^{-\frac{1}{2}} \boldsymbol{b} \\
\hat{\boldsymbol{c}}=\boldsymbol{c} \boldsymbol{K}_{c}^{\frac{1}{2}} \hat{\boldsymbol{T}}^{T}, \quad \hat{\boldsymbol{W}}_{o}=\hat{\boldsymbol{T}} \boldsymbol{K}_{c}^{\frac{1}{2}} \boldsymbol{W}_{o} \boldsymbol{K}_{c}^{\frac{1}{2}} \hat{\boldsymbol{T}}^{T} .
\end{array}
$$

From the foregoing arguments, the problem of obtaining matrices $\boldsymbol{T}$ and $\boldsymbol{D}_{1}, \boldsymbol{D}_{2}, \cdots, \boldsymbol{D}_{N}$ that jointly minimize (14) subject to the $l_{2}$-scaling constraints in (13) is now converted into an unconstrained optimization problem of obtaining $\hat{T}$ and $\boldsymbol{D}_{1}, \boldsymbol{D}_{2}, \cdots, \boldsymbol{D}_{N}$ that jointly minimize $J(\hat{\boldsymbol{T}}, \boldsymbol{D})$ in (18).

Let $\boldsymbol{x}$ be the column vector that collects the variables in matrices $\left[\boldsymbol{t}_{1}, \boldsymbol{t}_{2}, \cdots, \boldsymbol{t}_{n}\right]$ and $\boldsymbol{D}_{1}, \boldsymbol{D}_{2}, \cdots, \boldsymbol{D}_{N}$. Then $J(\hat{\boldsymbol{T}}, \boldsymbol{D})$ in (18) is a function of $\boldsymbol{x}$, denoted by $J(\boldsymbol{x})$. The proposed algorithm starts with an initial point $\boldsymbol{x}_{0}$ obtained from an initial assignment $\hat{\boldsymbol{T}}=\boldsymbol{D}_{1}=\boldsymbol{D}_{2}=\cdots=\boldsymbol{D}_{N}=\boldsymbol{I}_{n}$. In the $k$ th iteration, a quasi-Newton algorithm updates the most recent point $\boldsymbol{x}_{k}$ to point $\boldsymbol{x}_{k+1}$ as [21]

$$
\boldsymbol{x}_{k+1}=\boldsymbol{x}_{k}+\alpha_{k} \boldsymbol{d}_{k},
$$

where

$$
\begin{aligned}
\boldsymbol{d}_{k} & =-\boldsymbol{S}_{k} \nabla J\left(\boldsymbol{x}_{k}\right), \quad \alpha_{k}=\arg \left[\min _{\alpha} J\left(\boldsymbol{x}_{k}+\alpha \boldsymbol{d}_{k}\right)\right] \\
\boldsymbol{S}_{k+1} & =\boldsymbol{S}_{k}+\left(1+\frac{\boldsymbol{\gamma}_{k}^{T} \boldsymbol{S}_{k} \boldsymbol{\gamma}_{k}}{\boldsymbol{\gamma}_{k}^{T} \boldsymbol{\delta}_{k}}\right) \frac{\boldsymbol{\delta}_{k} \boldsymbol{\delta}_{k}^{T}}{\boldsymbol{\gamma}_{k}^{T} \boldsymbol{\delta}_{k}}-\frac{\boldsymbol{\delta}_{k} \boldsymbol{\gamma}_{k}^{T} \boldsymbol{S}_{k}+\boldsymbol{S}_{k} \boldsymbol{\gamma}_{k} \boldsymbol{\delta}_{k}^{T}}{\boldsymbol{\gamma}_{k}^{T} \boldsymbol{\delta}_{k}} \\
\boldsymbol{S}_{0} & =\boldsymbol{I}, \quad \boldsymbol{\delta}_{k}=\boldsymbol{x}_{k+1}-\boldsymbol{x}_{k}, \quad \boldsymbol{\gamma}_{k}=\nabla J\left(\boldsymbol{x}_{k+1}\right)-\nabla J\left(\boldsymbol{x}_{k}\right) .
\end{aligned}
$$


Here, $\nabla J(\boldsymbol{x})$ is the gradient of $J(\boldsymbol{x})$ with respect to $\boldsymbol{x}$, and $\boldsymbol{S}_{k}$ is a positive-definite approximation of the inverse Hessian matrix of $J\left(\boldsymbol{x}_{k}\right)$. This iteration process continues until

$$
\left|J\left(\boldsymbol{x}_{k+1}\right)-J\left(\boldsymbol{x}_{k}\right)\right|<\varepsilon
$$

where $\varepsilon>0$ is a prescribed tolerance.

In what follows, we derive closed-form expressions of $\nabla J(\boldsymbol{x})$ for the cases where $\boldsymbol{D}_{1}, \boldsymbol{D}_{2}, \cdots, \boldsymbol{D}_{N}$ assume the form of diagonal or scalar matrices.

Case 1: $\boldsymbol{D}_{p}=\operatorname{diag}\left\{d_{p 1}, d_{p 2}, \cdots, d_{p n}\right\}$ for $p=1,2, \cdots, N$ In this case, it follows that

$$
\begin{gathered}
\frac{\partial J(\boldsymbol{x})}{\partial t_{i j}}=2 \boldsymbol{e}_{j}^{T}\left[\hat{\boldsymbol{A}}^{T} \hat{\boldsymbol{W}}_{o} \hat{\boldsymbol{A}}-\sum_{p=1}^{N}\left(\hat{\boldsymbol{W}}_{o} \hat{\boldsymbol{A}}^{p}+\left(\hat{\boldsymbol{A}}^{T}\right)^{p} \hat{\boldsymbol{W}}_{o}\right) \boldsymbol{D}_{p}\right. \\
\left.+\frac{1}{2} \sum_{p=1}^{N} \sum_{q=1}^{N}\left(\hat{\boldsymbol{W}}_{o} \hat{\boldsymbol{A}}^{|p-q|}+\left(\hat{\boldsymbol{A}}^{T}\right)^{|p-q|} \hat{\boldsymbol{W}}_{o}\right) \boldsymbol{D}_{p} \boldsymbol{D}_{q}\right] \hat{\boldsymbol{T}} \boldsymbol{g}_{i j} \\
i, j=1,2, \cdots, n
\end{gathered}
$$

where

$$
\boldsymbol{g}_{i j}=\partial\left\{\frac{\boldsymbol{t}_{j}}{\left\|\boldsymbol{t}_{j}\right\|}\right\} / \partial t_{i j}=\frac{1}{\left\|\boldsymbol{t}_{j}\right\|^{3}}\left(t_{i j} \boldsymbol{t}_{j}-\left\|\boldsymbol{t}_{j}\right\|^{2} \boldsymbol{e}_{i}\right) .
$$

Moreover,

$$
\begin{gathered}
\frac{\partial J(\boldsymbol{x})}{\partial d_{p i}}=-2\left(\hat{\boldsymbol{W}}_{o} \hat{\boldsymbol{A}}^{p}\right)_{i i}+2 \sum_{q=1}^{N} d_{q i}\left(\hat{\boldsymbol{W}}_{o} \hat{\boldsymbol{A}}^{|p-q|}\right)_{i i} \\
p=1,2, \cdots, N ; \quad i=1,2, \cdots, n .
\end{gathered}
$$

Case 2: $\boldsymbol{D}_{p}=\alpha_{p} \boldsymbol{I}_{n}$ for $p=1,2, \cdots, N$

The gradient of $J(\boldsymbol{x})$ can be calculated as

$$
\begin{gathered}
\frac{\partial J(\boldsymbol{x})}{\partial t_{i j}}=2 \boldsymbol{e}_{j}^{T}\left[\hat{\boldsymbol{A}}^{T} \hat{\boldsymbol{W}}_{o} \hat{\boldsymbol{A}}-\sum_{p=1}^{N} \alpha_{p}\left(\hat{\boldsymbol{W}}_{o} \hat{\boldsymbol{A}}^{p}+\left(\hat{\boldsymbol{A}}^{T}\right)^{p} \hat{\boldsymbol{W}}_{o}\right)\right. \\
\left.+\frac{1}{2} \sum_{p=1}^{N} \sum_{q=1}^{N} \alpha_{p} \alpha_{q}\left(\hat{\boldsymbol{W}}_{o} \hat{\boldsymbol{A}}^{|p-q|}+\left(\hat{\boldsymbol{A}}^{T}\right)^{|p-q|} \hat{\boldsymbol{W}}_{o}\right)\right] \hat{\boldsymbol{T}} \boldsymbol{g}_{i j} \\
\frac{\partial J(\boldsymbol{x})}{\partial \alpha_{p}}=-2 \operatorname{tr}\left(\hat{\boldsymbol{W}}_{o} \hat{\boldsymbol{A}}^{p}\right)+2 \sum_{q=1}^{N} \alpha_{q} \operatorname{tr}\left(\hat{\boldsymbol{W}}_{o} \hat{\boldsymbol{A}}^{|p-q|}\right) \\
i, j=1,2, \cdots, n ; \quad p=1,2, \cdots, N .
\end{gathered}
$$

\section{AN ILLUSTRATIVE EXAMPLE}

As a numerical example, consider a state-space digital filter $(\boldsymbol{A}, \boldsymbol{b}, \boldsymbol{c}, d)_{3}$ described by

$$
\begin{aligned}
\boldsymbol{A} & =\left[\begin{array}{ccc}
0 & 1 & 0 \\
0 & 0 & 1 \\
0.453770 & -1.556160 & 1.974860
\end{array}\right] \\
\boldsymbol{b} & =\left[\begin{array}{lrc}
0 & 0 & 0.2420961
\end{array}\right]^{T} \\
\boldsymbol{c} & =\left[\begin{array}{lll}
0.095706 & 0.095086 & 0.327556
\end{array}\right] \\
d & =0.015940 .
\end{aligned}
$$

The controllability and observability Gramians $\boldsymbol{K}_{c}$ and $\boldsymbol{W}_{o}$ of the above filter were computed from (10) and (8) as

$$
\begin{aligned}
\boldsymbol{K}_{c}= & {\left[\begin{array}{rrr}
1.000000 & 0.872501 & 0.562822 \\
0.872501 & 1.000000 & 0.872501 \\
0.562822 & 0.872501 & 1.000000
\end{array}\right] } \\
\boldsymbol{W}_{o}= & {\left[\begin{array}{rrr}
0.820742 & -2.035323 & 1.628159 \\
-2.035323 & 5.307270 & -4.264912 \\
1.628159 & -4.264912 & 3.941488
\end{array}\right] . }
\end{aligned}
$$

The noise gain of the filter with no error feedforward and no EF was then computed from (9) as $J_{e 1}(\mathbf{0}, \mathbf{0})=10.069510$.

Case 1: $\boldsymbol{D}_{1}$ and $\boldsymbol{D}_{2}$ are diagonal matrices where $N=2$

The quasi-Newton algorithm was applied to minimize (18) with tolerance $\varepsilon=10^{-8}$ in (20). It took the algorithm 44 iterations to converge to the solution

$$
\begin{aligned}
\hat{\boldsymbol{T}} & =\left[\begin{array}{rrr}
1.444866 & -0.214988 & 0.437178 \\
-1.309716 & 1.306211 & 0.465756 \\
-0.577484 & 0.237097 & 1.275346
\end{array}\right] \\
\boldsymbol{D}_{1} & =\operatorname{diag}\left\{\begin{array}{lll}
0.6405 & 1.2864 & 1.44310
\end{array}\right\} \\
\boldsymbol{D}_{2} & =\operatorname{diag}\left\{\begin{array}{lll}
-0.0242 & -0.6992 & -0.6984
\end{array}\right\}
\end{aligned}
$$

with the noise gain $J(\hat{\boldsymbol{T}}, \boldsymbol{D})=0.003552$. The profile of $J(\hat{\boldsymbol{T}}, \boldsymbol{D})$ during the first 44 iterations of the algorithm is dipicted in Fig. 1.

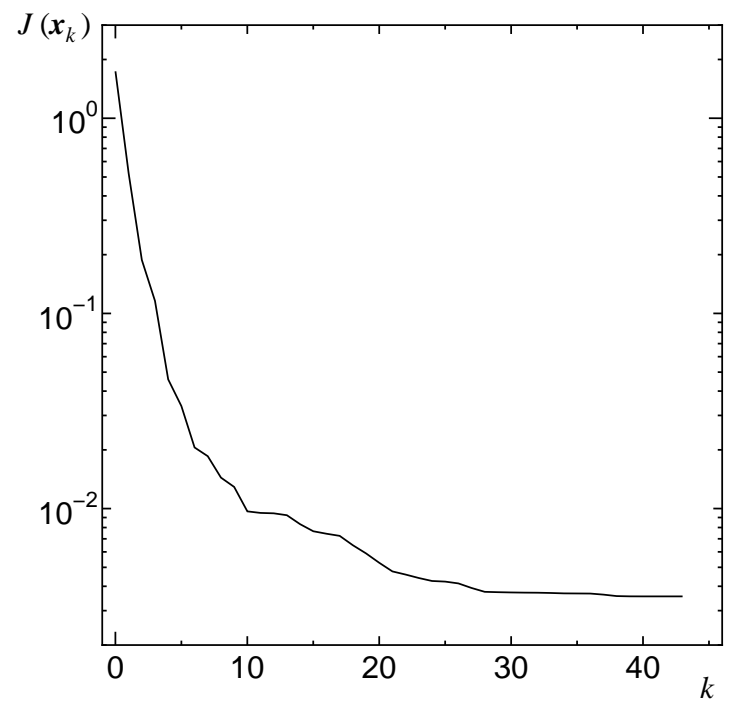

Fig. 1. Profile of $J(\hat{\boldsymbol{T}}, \boldsymbol{D})$ during the first 44 iterations.

Case 2: $\boldsymbol{D}_{1}$ and $\boldsymbol{D}_{2}$ are scalar matrices where $N=2$

The quasi-Newton algorithm with $\varepsilon=10^{-8}$ was applied to minimize (18) for scalar high-order EF matrices $\boldsymbol{D}_{1}$ and $\boldsymbol{D}_{2}$. It took the algorithm 19 iterations to converge to the solution

$$
\begin{aligned}
\hat{\boldsymbol{T}} & =\left[\begin{array}{rrr}
1.219422 & -0.555940 & -0.042678 \\
-0.434261 & 1.235725 & 0.697171 \\
-0.079476 & -0.077024 & 1.153868
\end{array}\right] \\
\boldsymbol{D}_{1} & =1.1973 \boldsymbol{I}_{3},
\end{aligned}
$$


with the noise gain $J(\hat{\boldsymbol{T}}, \boldsymbol{D})=0.050379$. The profile of $J(\hat{\boldsymbol{T}}, \boldsymbol{D})$ during the first 19 iterations of the algorithm is shown in Fig. 2.

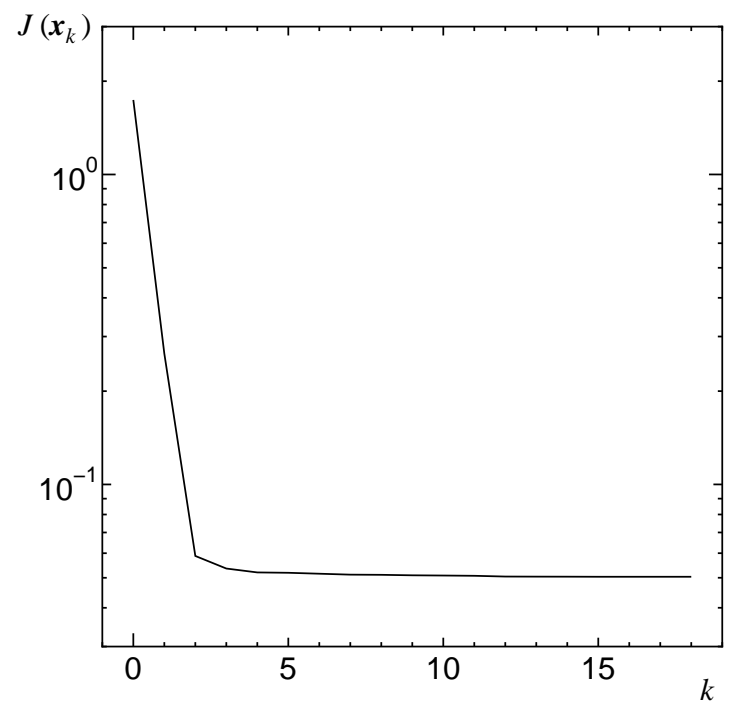

Fig. 2. Profile of $J(\hat{\boldsymbol{T}}, \boldsymbol{D})$ during the first 19 iterations.

The other results regarding the noise gain $J(\hat{\boldsymbol{T}}, \boldsymbol{D})$ in (18) were summarized in Table I where "Infinite Precision" shows the value of $J(\hat{\boldsymbol{T}}, \boldsymbol{D})$ derived from the optimal $\hat{\boldsymbol{T}}$ and $\boldsymbol{D}$, and "3-Bit Quantization" means that of $J(\hat{\boldsymbol{T}}, \boldsymbol{D})$ where only each entry of the optimal matrix $\boldsymbol{D}=\left[\boldsymbol{D}_{1}, \boldsymbol{D}_{2}\right]$ was rounded to a power-of-two representation with 3 bits after the binary point.

TABLE I

PERFORMANCE COMPARISON

\begin{tabular}{c|c|c|c}
\hline$N$ & $\boldsymbol{D}_{1}, \boldsymbol{D}_{2}, \cdots, \boldsymbol{D}_{N}$ & $\begin{array}{c}\text { Infinite } \\
\text { Precision }\end{array}$ & $\begin{array}{c}\text { 3-Bit } \\
\text { Quantization }\end{array}$ \\
\hline \hline \multirow{2}{*}{1} & Diagonal & 0.049981 & 0.083396 \\
\cline { 2 - 4 } & Scalar & 0.092729 & 0.105829 \\
\hline \multirow{2}{*}{2} & Diagonal & 0.003552 & 0.008444 \\
\cline { 2 - 4 } & Scalar & 0.050379 & 0.060035 \\
\hline
\end{tabular}

The results reported above have clearly demonstrated that the use of high-order (i.e. $N>1$ ) EF, when jointly optimized with state-space realization, can improve the performance of roundoff noise reduction in a significant manner.

\section{CONCLUSION}

The joint optimization problem of high-order EF and realization to minimize the effects of roundoff noise of statespace digital filters subject to $l_{2}$-scaling constraints has been investigated. It has been shown that the problem at hand can be converted into an unconstrained optimization problem by using linear algebraic techniques. Closed-form formulas for fast evaluation of the gradient of the objective function have been derived. An efficient quasi-Newton algorithm has been employed to solve the unconstrained optimization problem.
The proposed technique has been applied to the cases where the high-order EF has diagonal or scalar matrices. A numerical example has demonstrated the effectiveness of the proposed technique.

\section{REFERENCES}

[1] H. A. Spang, III and P. M. Shultheiss, "Reduction of quantizing noise by use of feedback," IRE Trans. Commun. Syst., vol. CS-10, pp. 373-380, Dec. 1962.

[2] T. Thong and B. Liu, "Error spectrum shaping in narrowband recursive digital filters," IEEE Trans. Acoust. Speech, Signal Processing, vol. 25, pp. 200-203, Apr. 1977.

[3] T. L. Chang and S. A. White, "An error cancellation digital filter structure and its distributed-arithmetic implementation," IEEE Trans. Circuits Syst., vol. 28, pp. 339-342, Apr. 1981.

[4] D. C. Munson and D. Liu, "Narrowband recursive filters with error spectrum shaping," IEEE Trans. Circuits Syst., vol. 28, pp. 160-163, Feb. 1981.

[5] W. E. Higgins and D. C. Munson, "Noise reduction strategies for digital filters: Error spectrum shaping versus the optimal linear state-space formulation," IEEE Trans. Acoust. Speech, Signal Processing, vol. 30, pp. 963-973, Dec. 1982.

[6] M. Renfors, "Roundoff noise in error-feedback state-space filters," Proc. Int. Conf. Acoustics, Speech, Signal Processing (ICASSP'83), pp. 619622, Apr. 1983.

[7] W. E. Higgins and D. C. Munson, "Optimal and suboptimal errorspectrum shaping for cascade-form digital filters," IEEE Trans. Circuits Syst., vol. 31, pp. 429-437, May 1984.

[8] T. I. Laakso and I. O. Hartimo, "Noise reduction in recursive digital filters using high-order error feedback," IEEE Trans. Signal Processing, vol. 40, pp. 1096-1107, May 1992

[9] P. P. Vaidyanathan, "On error-spectrum shaping in state-space digital filters," IEEE Trans. Circuits Syst., vol. 32, pp. 88-92, Jan. 1985.

[10] D. Williamson, "Roundoff noise minimization and pole-zero sensitivity in fixed-point digital filters using residue feedback," IEEE Trans. Acoust., Speech, Signal Processing, vol. 34, pp. 1210-1220, Oct. 1986.

[11] G. Li and M. Gevers, "Roundoff noise minimization using delta-operator realizations," IEEE Trans. Signal Processing, vol. 41, pp. 629-637, Feb. 1993.

[12] D. Williamson, "Delay replacement in direct form structures", IEEE Trans. Acoust., Speech, Signal Processing, vol. 36, pp. 453-460, Apr. 1988.

[13] M. M. Ekanayake and K. Premaratne, "Two-dimensional delta-operator formulated discrete-time systems: Analysis and synthesis of minimum roundoff noise realizations," Proc. IEEE Int. Symp. Circuits Syst. (ISCAS'96), vol. 2, pp. 213-216, May 1996.

[14] G. Li and Z. Zhao, "On the generalized DFIIt structure and its statespace realization in digital filter implementation," IEEE Trans. Circuits Syst. I, vol. 51, pp. 769-778, Apr. 2004.

[15] S. Y. Hwang, "Roundoff noise in state-space digital filtering: A general analysis," IEEE Trans. Acoust., Speech, Signal Processing, vol. 24, pp. 256-262, June 1976.

[16] C. T. Mullis and R. A. Roberts, "Synthesis of minimum roundoff noise fixed-point digital filters," IEEE Trans. Circuits Syst., vol. 23, pp. 551562, Sept. 1976.

[17] S. Y. Hwang, "Minimum uncorrelated unit noise in state-space digital filtering," IEEE Trans. Acoust., Speech, Signal Processing, vol. 25, pp. 273-281, Aug. 1977.

[18] L. B. Jackson, A. G. Lindgren and Y. Kim, "Optimal synthesis of second-order state-space structures for digital filters," IEEE Trans. Circuits Syst. , vol. 26, pp. 149-153, Mar. 1979.

[19] T. Hinamoto, H. Ohnishi and W.-S. Lu, "Roundoff noise minimization of state-space digital filters using separate and joint error feedback/coordinate transformation," IEEE Trans. Circuits Syst. I, vol. 50, pp. 23-33, Jan. 2003.

[20] W.-S. Lu and T. Hinamoto, "Jointly optimized error-feedback and realization for roundoff noise minimization in state-space digital filters," IEEE Trans. Signal Processing, vol. 53, pp. 2135-2145, June 2005.

[21] R. Fletcher, Practical Methods of Optimization, 2nd ed. Wiley, New York, 1987. 